\title{
Vocal fold paralysis following fine needle aspiration of the thyroid gland complicated by a neck hematoma
}

\author{
Zidan Mahmood Z ${ }^{1}$, Adi Brenner ${ }^{2}$, Narin Nard Carmel ${ }^{1}$ and Yael Oestreicher-Kedem ${ }^{1 *}$ \\ ${ }^{1}$ Departments of Otolaryngology Head \& Neck Surgery and Maxillofacial Surgery, Tel-Aviv University, Tel Aviv, Israel \\ ${ }^{2}$ Department of Radiology, Tel Aviv Sourasky Medical Center, Sackler School of Medicine, Tel-Aviv University, Tel Aviv, Israel
}

\begin{abstract}
\section{Introduction}

Fine needle aspiration (FNA) is usually the first step in the investigation of thyroid nodules [1]. It is a simple and inexpensive procedure [2], with high sensitivity and specificity for detection of malignancy [3,4]. It is also associated with few complications. We present a rare case of vocal fold paralysis (VFP) following FNA of a thyroid nodule complicated by a neck hematoma.
\end{abstract}

Objective: To describe the mechanism and clinical course of vocal fold paralysis (VFP) following fine needle aspiration (FNA) of the thyroid gland.

Case report: An 80-year old female presented to the emergency room due to hoarseness, dyspnea, and neck swelling. Her history was significant for FNA of the thyroid gland eight days earlier. Physical examination revealed left VFP and a left neck mass. Computed tomography of the neck was suggestive of a left neck hematoma. She was treated conservatively and her pain and neck swelling subsided gradually. Good left vocal movement was observed four months later.

Conclusion: VFP is a rare complication of FNA of the thyroid gland. Physicians performing this procedure should be aware of this complication. We suggest that this sequela be discussed with the patient prior to the procedure and be included in the informed patient consent form.

\section{Case report}

An 80-year-old woman presented to the emergency room due to hoarseness, cough, dyspnea, odynophagia and swelling of the left lateral neck. She had undergone an FNA of a left thyroid gland nodule eight days earlier. She had had several FNAs of the thyroid gland in the past, all of which, but the last FNA that was painful with repeated passes, were uneventful and showed benign cytological features. Her past medical history was significant for thyroid nodules, hypertension, dyslipidemia and type II diabetes mellitus. She denied the use of anticoagulant and anti-platelet medications.

The patient was stable with blood pressure of $163 / 87 \mathrm{mmHg}, \mathrm{O}_{2}$ saturation $98 \%$ and temperature of $36.7^{\circ} \mathrm{C}$. Physical examination revealed a tender swelling of the left lateral neck, without redness or warmth. Fiberoptic laryngoscopy revealed left VFP at a para-median position. The rest of the head and neck exam was unremarkable, and the results of a complete blood count, prothrombin time and partial thromboplastin time were within normal limits.

Computed tomography (CT) scan of the neck revealed left pyriform sinus dilatation and medial positioning and thickening of the left vocal fold (Figure 1A) at the supraglottic level, and medialization and thickening of the left vocal fold at the glottic level (Figure 1B). At the lower neck level, there was a hypo/isodense mass measuring 48X65 $\mathrm{mm}$ in the coronal plane, extending from the level of the left lobe of the thyroid gland to the upper mediastinum, and shifting the trachea, esophagus, left common carotid artery and jugular vein (Figures 2 and 3A-B). The inhomogeneous-looking left lobe of the thyroid was compressed by the mass which had a consistency that could represent a hematoma.

The patient was admitted to the otolaryngology department and treated with antibiotics and analgesics. She was discharged five days later when her pain and neck swelling had subsided, although the hoarseness persisted. Cytological findings of the left thyroid FNA were consistent with a colloid nodule.

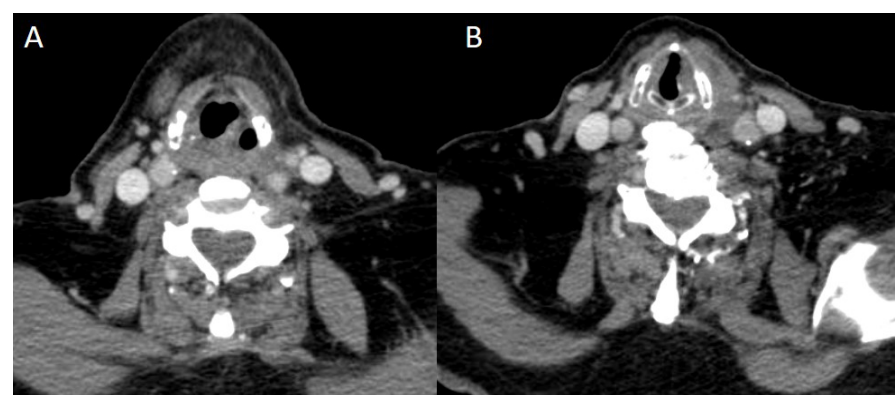

Figure 1. A CT scan: axial view of the neck at the supraglottic level. Note left pyriform sinus dilatation and medial positioning and thickening of the left aryepiglottic fold. These findings suggest left VFP. B CT scan: axial view of the neck at the glottic level. Note medialization and thickening of the left vocal fold. These finding suggest left VFP.

Correspondence to: Yael Oestreicher-Kedem, Department of Otolaryngology, Head \& Neck Surgery and Maxillofacial Surgery, Tel-Aviv Sourasky Medical Center 6 Weizman St. Tel-Aviv, Israel, Tel: +972-52-6212179; Fax: +972-36052905; E-mail: dkyo@013.net

Key words: fine needle aspiration, thyroid, vocal fold paralysis, hematoma

Received: August 20, 2017; Accepted: September 23, 2017; Published: September 25, 2017 


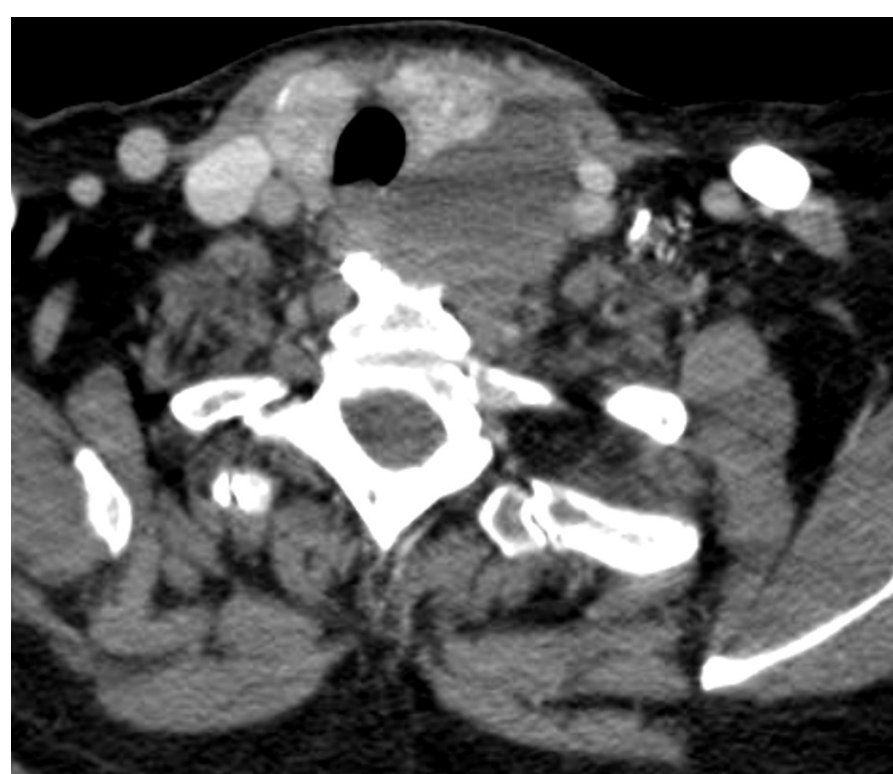

Figure 2. CT scan: axial view of the neck at the level of the thyroid gland. A mass is visible lateral to the left lobe of the thyroid, shifting it forward and causing a deviation of the trachea and esophagus to the right.

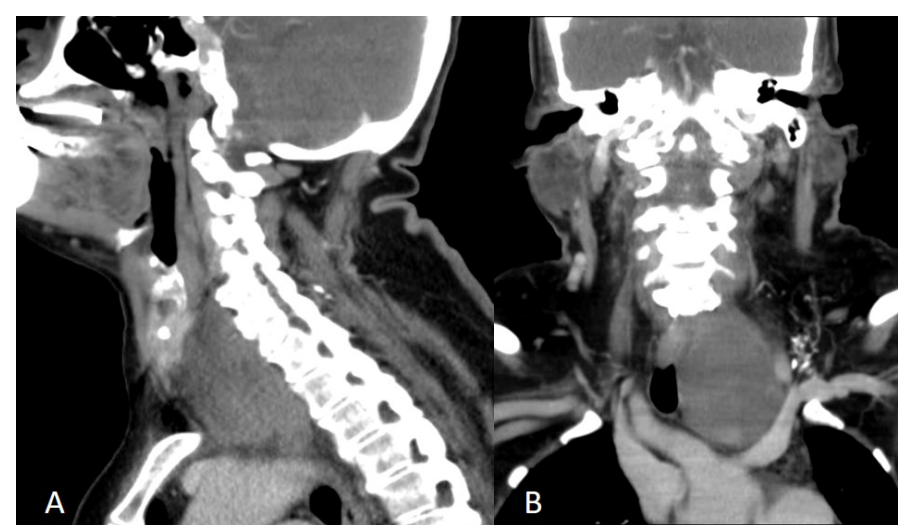

Figure 3. A Sagittal and $\mathbf{B}$ coronal CT scan views of the lower neck. The same mass as in Figure 2 is seen extending from the level of the left thyroid gland to the upper mediastinum.

She was followed monthly at the outpatient laryngology clinic. At the 3-month follow-up, she was still hoarse, and fiberoptic laryngoscopy revealed left VFP. A follow-up CT scan of the neck revealed only slight improvement in mass reduction compared to the initial CT scan. An ultrasound-guided FNA biopsy from the left neck mass was performed and the aspirate was sent for cytological exam. The cytological findings were consistent with an "old" hematoma. Four months after the initial FNA, the patient reported that her voice was "back to normal". The laryngeal examination revealed satisfactory movement of the left vocal cord with intact glottis closure.

\section{Discussion}

The herein described patient presented with new-onset VFP and neck swelling. The CT scan showed findings characteristic of VFP consisting of ipsilateral pyriform sinus dilatation, medial positioning and thickening of the ipsilateral aryepiglottic fold and ipsilateral vocal fold medialization and thickening [5].

FNA of the thyroid gland is rarely associated with complications [6]. Polyzos and Anastasilakis's systematic review ${ }^{2}$ summarized these rare sequelae, including: hematomas, infection, recurrent laryngeal nerve
(RLN) palsy, tracheal puncture, needle track seeding, needle track sinus and post-aspiration thyrotoxicosis. We now describe VFP after FNA of a thyroid nodule. This complication is rare, with a reported incidence of $0.036 \%$ [1]. The actual incidence is unknown because asymptomatic patients do not routinely undergo laryngoscopy. The exact mechanism of VFP after FNA of the thyroid gland is also unknown. Proposed mechanisms include:[1,2,7]

1. Stretching of the nerve over a thyroid hematoma.

2. Pressure on the recurrent laryngeal nerve by the hematoma against the trachea.

3. Inflammation around the nerve in the area of the FNA.

4. Thrombosis of minute arteries that constitute the vascular supply of the recurrent laryngeal nerve (RLN).

5. Direct injury of the nerve by the biopsy needle.

We assume that the mechanism in our case was stretching or pressure over the left RLN by the hematoma. It is essential for doctors performing thyroid FNAs to be alert to possible risk factors for thyroid hemorrhage and hematoma, such as multiple needle passes, patient use of anti-platelet or anti-coagulant medications and medical conditions predisposing the patient to hemorrhage. Precautionary measures should also be taken for high-risk patients by using a thinner needle, making fewer passes, performing a less aggressive aspiration, and extending the time of supervision following the procedure [6].

In the setting of malignant thyroid disease, VFP in a patient who underwent prior FNA of the thyroid gland without documentation of vocal fold movement impairment may be a confounding factor. In these cases, the VFP may be associated with the malignant process and not related to the FNA, thus indicative of a more aggressive surgery [8]. Ideally, all patients undergoing FNA of the thyroid should undergo pre-FNA vocal fold examination, but the rarity of VFP after thyroid FNA would make such a recommendation impractical, especially in terms of cost.

Regardless of the mechanism of action, spontaneous recovery of post-FNA VFP is to be expected, usually within 6 months [1,2], and the voice can be expected to be restored to normal.

\section{Conclusion}

VFP associated with FNA is a rare complication of which radiologists and otolaryngologists should be aware. This sequela should probably be discussed with the patient when obtaining consent for FNA of the thyroid gland.

\section{Funding}

There are no sources of funding or financial support.

\section{Disclosures}

The authors have no conflicts of interest or financial ties to disclose.

\section{Acknowledgment}

Esther Eshkol is thanked for editorial assistance.

\section{References}

1. Tomoda C, Takamura Y, Ito Y, Miya A, Miyauchi A (2006) Transient vocal cord paralysis after fine-needle aspiration biopsy of thyroid tumor. Thyroid 16: 697-699. [Crossref]

2. Polyzos SA, Anastasilakis AD (2009) Clinical complications following thyroid fineneedle biopsy: a systematic review. Clin Endocrinol (Oxf) 71: 157-165. [Crossref] 
3. Okamoto T, Yamashita T, Harasawa A, Kanamuro T, Aiba M, et al. (1994) Test performances of three diagnostic procedures in evaluating thyroid nodules: physical examination, ultrasonography and fine needle aspiration cytology. Endocr J 41: 243247. [Crossref]

4. Carpi A, Ferrari E, De Gaudio C, Sagripanti A, Nicolini A, et al. (1994) The value of aspiration needle biopsy in evaluating thyroid nodules. Thyroidology 6: 5-9. [Crossref]

5. Chin SC, Edelstein S, Chen CY, Som PM (2003) Using CT to localize side and level of vocal cord paralysis. AJR Am J Roentgenol 180: 1165-1170. [Crossref]
6. Hor T, Lahiri SW (2008) Bilateral thyroid hematomas after fine-needle aspiration causing acute airway obstruction. Thyroid 18: 567-569. [Crossref]

7. Leung AM, Farwell AP (2008) Unsatisfactory consequences from fine-needle aspiration biopsy of thyroid nodules. Thyroid 18: 491-492. [Crossref]

8. Hulin SJ, Harris KP (2006) Thyroid fine needle cytology complicated by recurrent laryngeal nerve palsy and unnecessary radical surgery. J Laryngol Otol 20: 970-971. [Crossref]

Copyright: (C2017 Mahmood ZZ. This is an open-access article distributed under the terms of the Creative Commons Attribution License, which permits unrestricted use, distribution, and reproduction in any medium, provided the original author and source are credited. 